Network Working Group

Request for Comments: 3524

Category: Standards Track
G. Camarillo

A. Monrad

Ericsson

April 2003

\title{
Mapping of Media Streams to Resource Reservation Flows
}

Status of this Memo

This document specifies an Internet standards track protocol for the Internet community, and requests discussion and suggestions for improvements. Please refer to the current edition of the "Internet Official Protocol Standards" (STD 1) for the standardization state and status of this protocol. Distribution of this memo is unlimited.

Copyright Notice

Copyright (C) The Internet Society (2003). All Rights Reserved.

Abstract

This document defines an extension to the Session Description Protocol (SDP) grouping framework. It allows requesting a group of media streams to be mapped into a single resource reservation flow. The SDP syntax needed is defined, as well as a new "semantics" attribute called Single Reservation Flow (SRF).

Table of Contents

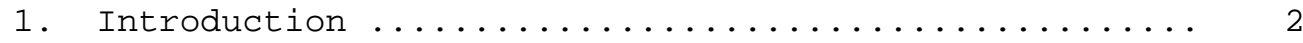

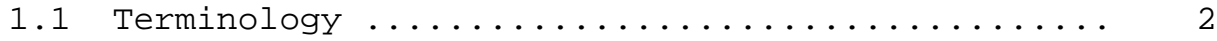

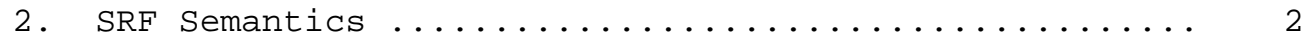

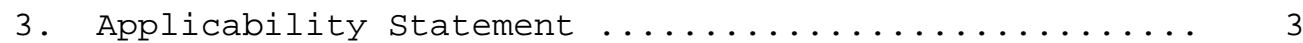

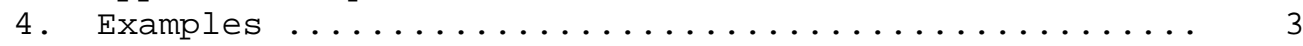

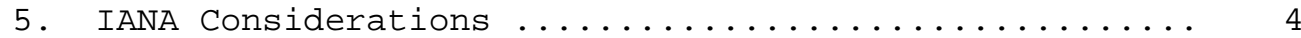

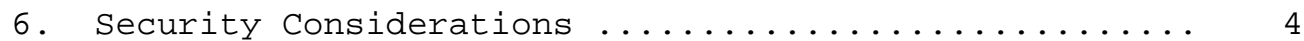

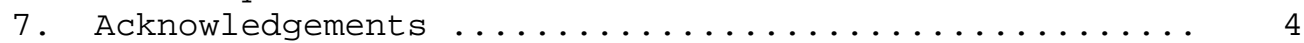

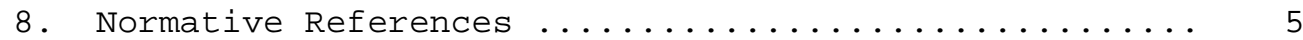

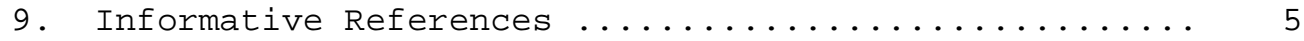

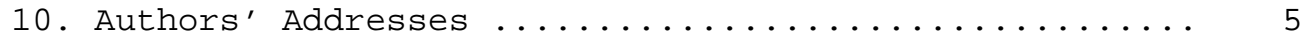

11. Full Copyright Statement ................. 6 


\section{Introduction}

Resource reservation protocols assign network resources to particular flows of IP packets. When a router receives an IP packet, it applies a filter in order to map the packet to the flow it belongs. The router provides the IP packet with the Quality of Service (QOS) corresponding to its flow. Routers typically use the source and the destination IP addresses and port numbers to filter packets.

Multimedia sessions typically contain multiple media streams (e.g. an audio stream and a video stream). In order to provide QoS for a multimedia session it is necessary to map all the media streams to resource reservation flows. This mapping can be performed in different ways. Two possible ways are to map all the media streams to a single resource reservation flow or to map every single media stream to a different resource reservation flow. Some applications require that the former type of mapping is performed while other applications require the latter. It is even possible that a mixture of both mappings is required for a particular media session. For instance, a multimedia session with three media streams might require that two of them are mapped into a single reservation flow while the third media stream uses a second reservation flow.

This document defines the SDP [1] syntax needed to express how media streams need to be mapped into reservation flows. For this purpose, we use the SDP grouping framework [2] and define a new "semantics" attribute called Single Reservation Flow (SRF).

1.1 Terminology

In this document, the key words "MUST", "MUST NOT", "REQUIRED", "SHALL", "SHALL NOT", "SHOULD", "SHOULD NOT", "RECOMMENDED", "MAY", and "OPTIONAL" are to be interpreted as described in BCP 14, RFC 2119 [3] and indicate requirement levels for compliant SIP implementations.

\section{SRF Semantics}

We define a new "semantics" attribute within the SDP grouping framework [2]: Single Reservation Flow (SRF).

Media lines grouped using SRF semantics SHOULD be mapped into the same resource reservation flow. Media lines that do not belong to a particular SRF group SHOULD NOT be mapped into the reservation flow used for that SRF group. 
RFC 3524 Mapping Media Streams to Resource Reservation Flows April 2003

Note that an SRF group MAY consist of a single media line. In that case, following the definition above, that media line will be mapped into one reservation flow. That reservation flow will carry traffic from that media line, and from no other media lines.

3. Applicability statement

The way resource reservation works in some scenarios makes it unnecessary to use the mechanism described in this document. Some resource reservation protocols allow the entity generating the SDP session description to allocate resources in both directions (i.e., sendrecv) for the session. In this case, the generator of the session description can chose any particular mapping of media flows and reservation flows.

The mechanism described in this document is useful when the remote party needs to be involved in the resource reservation.

4. Examples

For this example, we have chosen to use SIP [4] to transport SDP sessions and RSVP [5] to establish reservation flows. However, other protocols or mechanisms could be used instead without affecting the SDP syntax.

A user agent receives a SIP INVITE with the SDP below:

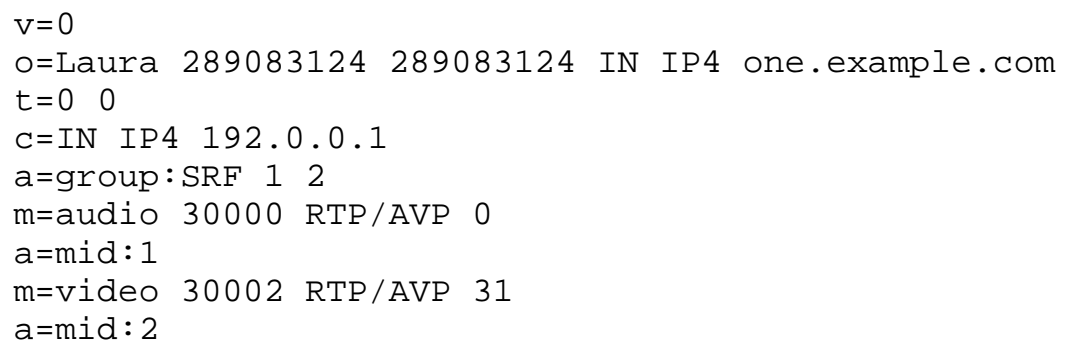

This user agent uses RSVP to perform resource reservation. Since both media streams are part of an SRF group, the user agent will establish a single RSVP session. An RSVP session is defined by the triple: (DestAddress, Protocolld[, DstPort]). Table 1 shows the parameters used to establish the RSVP session.

If the same user agent received an SDP session description with the same media streams but without the group line, it would be free to map the two media streams into two different RSVP sessions. 
RFC 3524 Mapping Media Streams to Resource Reservation Flows April 2003

\begin{tabular}{crcc} 
Session Number DestAddress & Protocolld & DstPort \\
\hline 1 & 192.0 .0 .1 & UDP & any \\
Table 1: Parameters needed to establish the RSVP session
\end{tabular}

5. IANA Considerations

IANA has registered the following new "semantics" attribute for the SDP grouping framework [2]. It has been registered in the SDP parameters registry (http://www.iana.org/assignments/sdp-parameters) under Semantics for the "group" SDP Attribute:

$\begin{array}{lcc}\text { Semantics } & \text { Token } & \text { Reference } \\ ---------------- & ----- & -------- \\ \text { Single Reservation flow } & \text { SRF } & \text { [RFC3524] }\end{array}$

6. Security Considerations

An attacker adding group lines using the SRF semantics to an SDP session description could force a user agent to establish a larger or a smaller number of resource reservation flows than needed. This could consume extra resources in the end-point or degrade the quality of service for a particular session. It is thus STRONGLY RECOMMENDED that integrity protection be applied to the SDP session descriptions. For session descriptions carried in SIP, S/MIME is the natural choice to provide such end-to-end integrity protection, as described in RFC 3261 [4]. Other applications MAY use a different form of integrity protection.

7. Acknowledgements

Jonathan Rosenberg provided useful comments about the applicability of the mechanism described in this document. 
RFC 3524 Mapping Media Streams to Resource Reservation Flows April 2003

8. Normative References

[1] Handley, M. and V. Jacobson, "SDP: Session Description Protocol", RFC 2327, April 1998.

[2] Camarillo, G., Eriksson, G., Holler, J. and H. Schulzrinne, "Grouping of Media Lines in the Session Description Protocol (SDP) ", December 2002 .

[3] Bradner, S., "Key words for use in RFCs to indicate requirement levels", BCP 14, RFC 2119, March 1997.

9. Informative References

[4] Rosenberg, J., Schulzrinne, H., Camarillo, G., Johnston, A., Peterson, J., Sparks, R., Handley, M. and E. Schooler, "SIP: Session Initiation Protocol", RFC 3261, June 2002.

[5] Braden, R., Zhang, L., Berson, S., Herzog, S. and S. Jamin, "Resource ReSerVation protocol (RSVP) -- Version 1 Functional Specification", RFC 2205, september 1997.

10. Authors' Addresses

Gonzalo Camarillo

Ericsson

Advanced Signalling Research Lab.

FIN-02420 Jorvas

Finland

EMail: Gonzalo.Camarillodericsson.com

Atle Monrad

Ericsson

N-4898 Grimstad

Norway

EMail: atle.monradeericsson.com 
11. Full Copyright statement

Copyright (C) The Internet Society (2003). All Rights Reserved.

This document and translations of it may be copied and furnished to others, and derivative works that comment on or otherwise explain it or assist in its implementation may be prepared, copied, published and distributed, in whole or in part, without restriction of any kind, provided that the above copyright notice and this paragraph are included on all such copies and derivative works. However, this document itself may not be modified in any way, such as by removing the copyright notice or references to the Internet society or other Internet organizations, except as needed for the purpose of developing Internet standards in which case the procedures for copyrights defined in the Internet Standards process must be followed, or as required to translate it into languages other than English.

The limited permissions granted above are perpetual and will not be revoked by the Internet society or its successors or assigns.

This document and the information contained herein is provided on an "AS IS" basis and THE INTERNET SOCIETY AND THE INTERNET ENGINEERING TASK FORCE DISCLAIMS ALL WARRANTIES, EXPRESS OR IMPLIED, INCLUDING BUT NOT LIMITED TO ANY WARRANTY THAT THE USE OF THE INFORMATION HEREIN WILL NOT INFRINGE ANY RIGHTS OR ANY IMPLIED WARRANTIES OF MERCHANTABILITY OR FITNESS FOR A PARTICULAR PURPOSE.

Acknowledgement

Funding for the RFC Editor function is currently provided by the Internet society. 